\title{
Simulation of SEP Routing Protocol and Compare with USEP
}

\author{
Surendra Verma ${ }^{1}$, Dr. R. K. Paliwal ${ }^{2}$, Dr. K. C. Mahajan ${ }^{3}$ \\ Research Scholar, Electronics \& Comm. Engg Department, Mewar University, Chittorgarh (Raj), India ${ }^{1}$ \\ Professor, Mewar University, Chittorgarh (Raj), India ${ }^{2}$ \\ Professor, Chouksey Engineering College Bilaspur (CG), India ${ }^{3}$
}

\begin{abstract}
A typical Wireless Sensor Networks is a multi-hop wireless network, is composed of large number of sensor devices. In case of routing protocols the wireless sensor network requirements are very specific, and it is the outcome of distributed nature and dynamic topology. There are many energy efficient algorithms which have been proposed in recent past. We may classify this algorithm on the basis of Reliable Routing, Network topology, Communication model and Network structure. We have analyzed and compared the performances of SEP and LEACH multiple routing protocols with our USEP on the basis of network lifetime and throughput.
\end{abstract}

Keywords: WSN, BS, SEP, USEP

\section{INTRODUCTION}

Today we are using huge number services using Wireless Sesnor Network(WSN). In year 80s, the primary Wireless Sensor Network has designed and developed by the military defence forces. It extensively used in several countries of the world for example Vietnam War has used some applications. WSN consist of wast number of mobile or stationary sensor nodes. We deployed power sensor node having low power and senses event data from the neighbor sensor node. Once it finds a secure connection through sensor nodes it may convey message to a base station or remote processing unit. Moreover, can be deployed in their environment randomly or with a proper deployment mechanism.

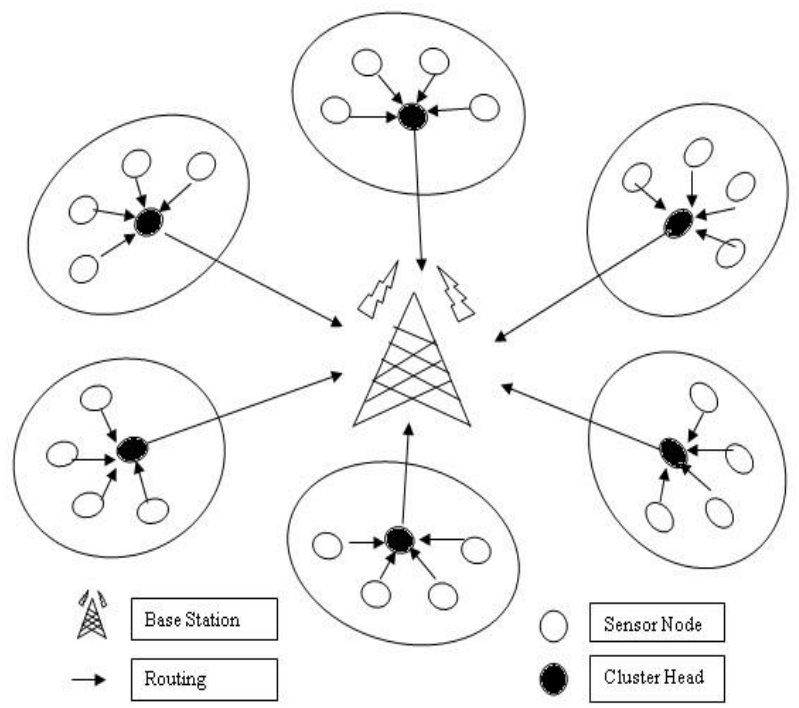

Figure No.1 A Basic Wireless Sensor Network with clustering

The figure No.1 shows the communication between the base stations by using less potential and higher bandwidth link. It shows that Sensor nodes are connected to its cluster head where an event is occur and the cluster head provide a communication link to the base station and its neighbour cluster head. The sensors detect events, which generated by a target/source node in the environment near the cluster head. If any node called sink want to some data from other node called source then sink communicates to its nearest cluster head. The head catches the event and report to the base station or the sink. The sensor nodes sense and detect events for the target node. After recognition of source node and sink node a link establishes by WSN. Now the communications occur between source node and sink node. 


\section{International Journal of Innovative Research in Electrical, Electronics, Instrumentation and Control Engineering}

Vol. 6, Issue 10, October 2018

\section{ROUTING ALGORITHMS}

A. Routing: Routing is the process in which a route from a source to a destination node is identified. It is achieved either by computing all routes before and pre-storing them or computing them when needed. Routing is used to provide a certain path between one node to another node for transmission.

B. SEP: SEP is stands for stable election protocol. The LEACH protocol has some problems like single hop, where any node can communicate directly to $\mathrm{CH}$ and sink node. The cluster heads are adopted casually. Thus there is a probability of all cluster heads will be determined in the similar region. In this technique, it uses the idea of dynamic clustering. Due to cluster changes, the unwanted overhead increase. The SEP routing protocol is projected for two level heterogeneity networks. Heterogeneity can be understood by the energy level, the protocol suggests two energy level cluster head in their cluster region. The nodes that ensure more energy level than the other nodes are called advance nodes and other nodes are the normal nodes. This protocol offer improved energy consumption, data delivery ratio, overhead and network life for WSN.

C. USEP: We propose an innovative routing algorithm USEP. USEP stand for Ultra stable Threshold sensitive election protocol. It is improvement version of ESEP. USEP is a reactive routing protocol and using five level heterogeneity.

According to probability the sensor nodes can select cluster heads at any time. The threshold level of channel head can be calculate with the help of formula given as

$$
\mathrm{Tn}=\mathrm{p} /[1-\mathrm{p}[\mathrm{r} \cdot \bmod (1 / \mathrm{p})]]
$$

The stable election protocol had started two type of heterogeneity. The probability can be calculated by equation 3 and 4. A segment of $m$ of $n$ number of nodes is delivered with an additional energy factor $\alpha$, which are called advanced nodes. So probabilities of normal and advanced nodes are

$$
\begin{aligned}
& \text { Pnnrm=popt }[1+\mathrm{m} \alpha] \\
& \text { Pnadv }=\operatorname{popt}(1+\alpha)[1+\mathrm{m} \alpha]
\end{aligned}
$$

Where popt is optional probability of each node to become $\mathrm{CH}$. When the padv or $\mathrm{m}$ will increases then the improve system performance parameter. We suggest popt and $\mathrm{m}$ should be correlated. For better explaining, we offer the types of sensor node with different energy stages. For multilevel heterogeneity, nodes with different energy levels are

1) Normal Nodes

2) Sub Normal Nodes

3) Intermediate Nodes

4) Advance Nodes

5) Super Nodes

The normal nodes have smallest energy and the Super nodes are having maximum energy level as compare to other. Remaining nodes have energy level as per order only. With the help of using segments of node, the intermediate nodes can be chosen. The intermediate node have energy is $\mu$ times more than by using below relationship of normal and sub normal nodes.

$$
\mathrm{E}_{\mathrm{ADV}}=\mathrm{E} 0(1+\alpha) \text {, is advance node energy, } \mathrm{E}_{\text {int }}=\mathrm{E} 0(1+\mu) \text { where } \mu=\alpha / 2 \text {, }
$$

So total energy of all these nodes will be $\eta \mathrm{b}(1+\alpha), \eta \mathrm{E} 0(1-\mathrm{m} \alpha-\mathrm{bn})$, and $\eta \mathrm{m} \mathrm{E} 0(1+\alpha), \eta \mathrm{E} 0(1+\mathrm{m} \alpha+\mathrm{bn}) \eta$ $\mathrm{E} 0(1+2 \mathrm{~m} \alpha+\mathrm{bn})$ respectively. Where $\mathrm{n}$ number of nodes, $\mathrm{b}$ is proportion to intermediate nodes and $\mathrm{m}$ is proportion to total number of nodes with energy more than rest of nodes of advanced nodes. The optimal probability of sensor nodes are energy basis and it is very useful to select a channel head. It can be determined through below equation for all the nodes

$$
\begin{aligned}
& \text { Pnnrm=popt }[1+\mathrm{m} \alpha+\mathrm{b} \mu] \\
& \text { Psubnrm=popt }(1+\mu)[1+\mathrm{m} \alpha+\mathrm{b} \mu] \\
& \text { Pint=popt }(1+2 \mu)[1+\mathrm{m} \alpha+\mathrm{b} \mu] \\
& \text { Padv=popt }(1+\alpha)[1+\mathrm{m} \alpha+\mathrm{b} \mu] \\
& \text { Psuper=popt }(1+2 \alpha)[1+\mathrm{m} \alpha+\mathrm{b} \mu]
\end{aligned}
$$

As we would like ensure that $\mathrm{CH}$ election is done in the same way, as we have assumed, which is threshold level, we have taken another parameter into consideration. The entire node produces randomly a number including the value 0 and 1 . The value will decide the performance of sensor node. The node will respond as a cluster head, when the attribute value is less than critical value. For all these categories we have separates formulas for the calculation of threshold depending on their probabilities, which are given below: 


\section{International Journal of Innovative Research in Electrical, Electronics, Instrumentation and Control Engineering}

Vol. 6, Issue 10, October 2018

Tnrm=Pnrm/[1-Prnm[r.mod(1/Pnrm) $]$

Tint=Pint/[1-Pint[r.mod (1/Pint)]

Tadv=Padv/[1-Padv[r.mod (1/Padv)]

Tsup=Psup/[1-Psup[r.mod (1/Psup)]

Tsubn=Psubn/[1-Psubn[r.mod (1/Psubn)]

Average total number of $\mathrm{CHs}$ per round will be:

$$
n 1-m-b P n r m+n \cdot b \cdot P i n t+n \cdot m \cdot P a d j+n(1+m+b)=n \cdot P o p t
$$

On simulation we can see the average number of cluster head is just like similar to SEP or LEACH protocol. Whereas, in our USEP routing protocol research that provide the best energy consumption and data deliver

\section{SIMULATION MODEL}

In our network scenario, we deployed 100 sensor nodes in the network topology at random. From our consideration, the base station situated centrally in entire area of 100x100 Meter. The entire node configures for USEP routing protocol for a mobile wireless sensor network, the USEP routing algorithm has been assign as its routing protocol. We performed simulations 3500 round for different values of $\alpha$ and $\mathrm{m}$ while keeping $\mathrm{b}$ constant that is 0.4 . For the first case $\alpha=2, \mathrm{~m}=0.1$, this is complete to survey change in network's strength, existence and throughput corresponding to enlarge the number of advance nodes and their energies. Since $\mathrm{P}_{\mathrm{opt}}=0.1$, is the optimal probability of CHs, by using equations (4), (5) and (6), (7) \& (8) we obtained different probabilities for each type of nodes in accordance with different values of $\alpha$ and $\mathrm{m}$. Other parameters used in simulations are given below in table no.1.

Table No. - 1 Parameter for SEP \& USEP

\begin{tabular}{|c|c|l|c|}
\hline S. No & Parameters & & Value \\
\hline 1 & $\mathrm{n}$ & Number of nodes & 100 \\
\hline 2 & $\mathrm{E}_{\mathrm{Elec}}$ & Energy dissipation to run & $50 \mathrm{~nJ} / \mathrm{bit}$ \\
\hline 3 & $\mathrm{Efs}$ & Free space model of transmitter amplifier & $10 \mathrm{pJ} / \mathrm{bit} / \mathrm{message}$ \\
\hline 3 & $\mathrm{E}_{\mathrm{DA}}$ & Data aggregation & $5 \mathrm{nj} / \mathrm{bit} / \mathrm{message}$ \\
\hline 4 & $\mathrm{E}_{\mathrm{MP}}$ & Multi-path model of transmitter amplifier & 0.0013 \\
\hline 5 & $\mathrm{E}$ & Initial energy of sensor nodes & $0.5 \mathrm{j}$ \\
\hline 7 & $\mathrm{P}_{\mathrm{Opt}}$ & Optical Probability & 0.1 \\
\hline 8 & $\mathrm{r}_{\max }$ & Simulation time & $3500 \mathrm{~ms}$ \\
\hline 9 & $\alpha$ & Energy Factor & 2 \\
\hline 10 & $\mathrm{~m}$ & Proportion of advanced nodes to $\mathrm{n}$ & 0.1 \\
\hline
\end{tabular}

IV. SIMULATION RESULT

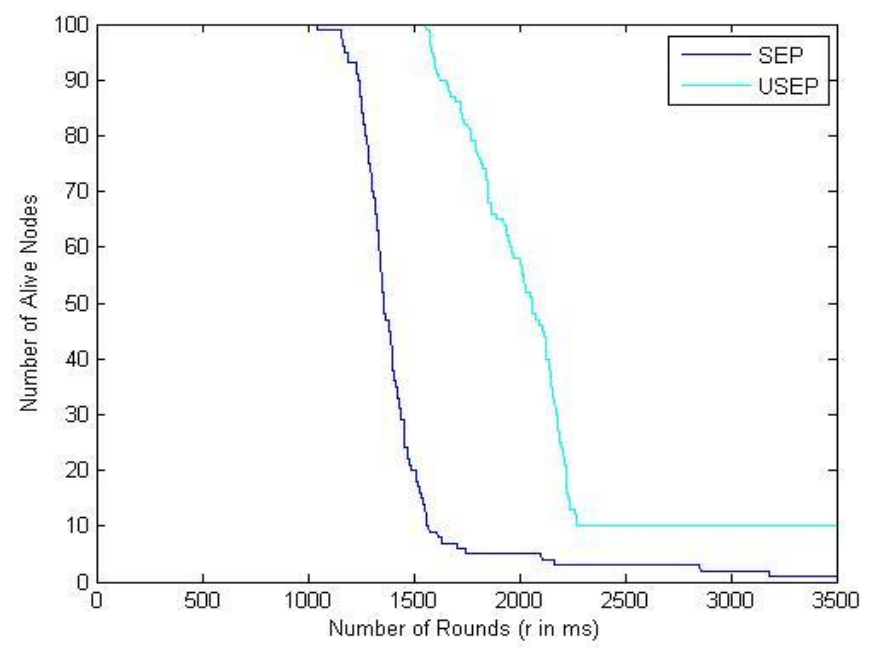

Figure No 2 Alive nodes for USEP and SEP 


\section{International Journal of Innovative Research in Electrical, Electronics, Instrumentation and Control Engineering}

Vol. 6, Issue 10, October 2018

Figure no 5.17 shows the Comparison of USEP and SEP routing algorithms for MWSN in terms of network life of the sensor nodes. We can see in the graph, number of round at $\mathrm{X}$-axis and Number of alive sensor node at $\mathrm{Y}$-axis. We choose 3500 rounds here to have clearer view.

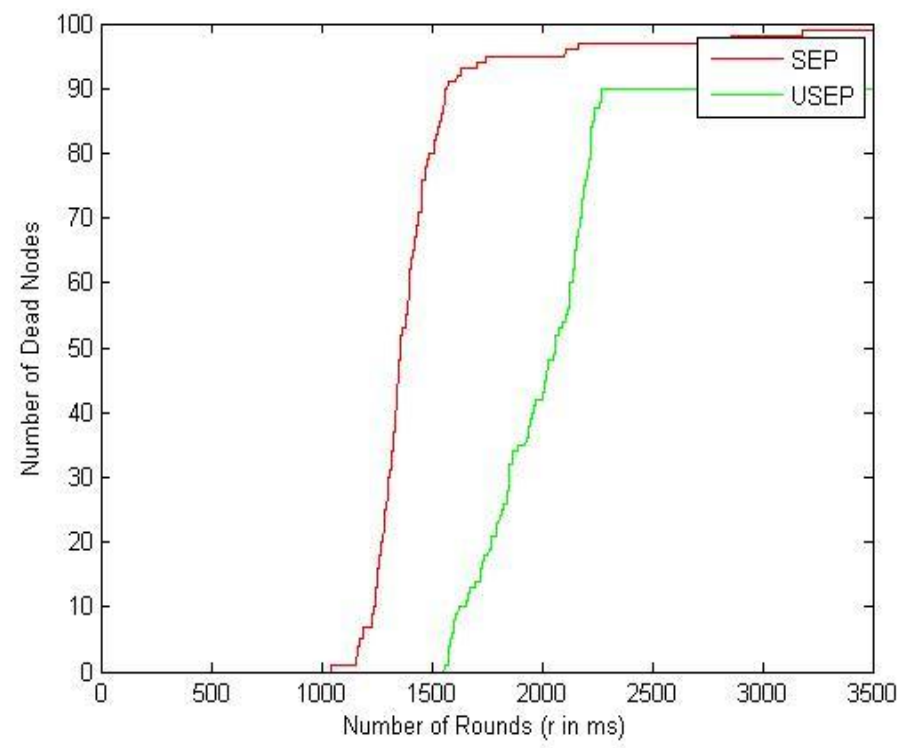

Figure No 3 Dead nodes for USEP and SEP

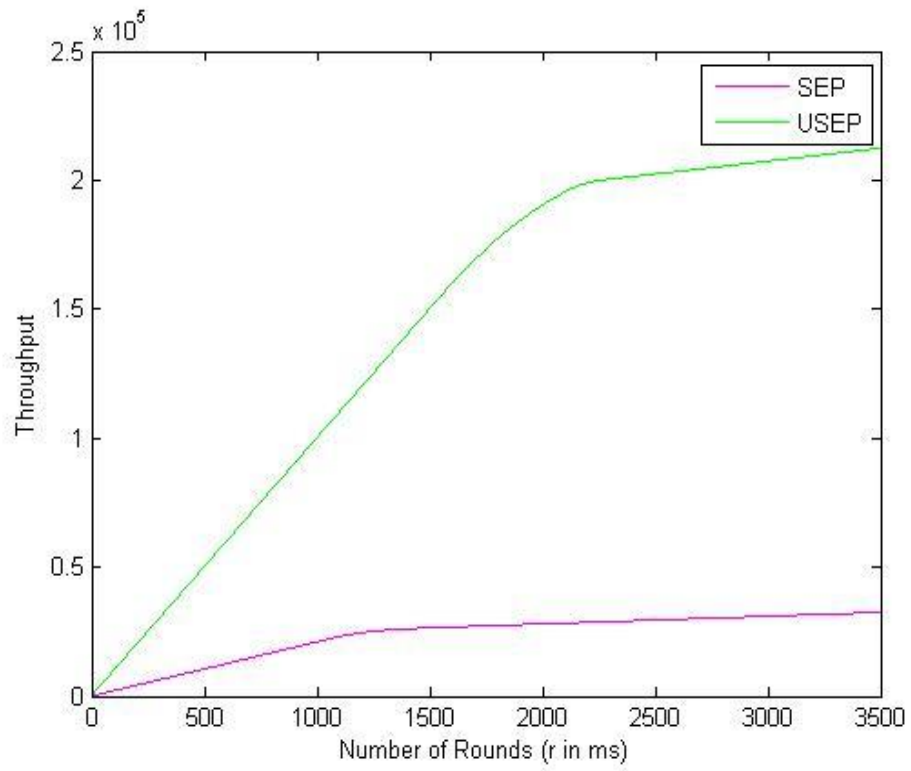

Figure No 4 Throughput for USEP and SEP

\section{CONCLUSION}

The simulation gives the knowledge how to use routing schemes in dynamic network. We offer a new energy efficient routing schemes for MWSN with mobile sensor nodes that have been obtainable in the literature survey. We have the universal goal of trying to enlarge the network life, throughput and energy consumption for the mobile sensor nodes. We Simulate SEP routing protocol and compared the performances with USEP on the basis of performance parameter like network life and throughput. The performance of USEP is improved as compared to SEP. On the basis of evaluation on MATLAB R2009a and R2013b simulator we clearly see that energy threshold and intelligent routing makes USEP a better approach for mobile routing choice. 


\title{
International Journal of Innovative Research in Electrical, Electronics, Instrumentation and Control Engineering
}

\author{
Vol. 6, Issue 10, October 2018
}

\section{REFERENCES}

[1]. Surendra Verma, Dr. K C Mahajan,” A Survey on Energy Efficient Routing Protocols for Wireless Sensor Networks \& Comparative analysis with USEP" SSRG International Journal of Electronics and Communication Engineering (SSRG-IJECE) - Volume 3 Issue 10 - October 2016.

[2]. Surendra Verma, Dr. K C Mahajan, "USEP: Ultra Stable Threshold Sensitive Election Protocol for Mobile WSN" International Journal of Computer Applications (0975 - 8887) Volume 100- No.3, August 2014

[3]. P. Saranya, Mrs. M. Parameswari," Preparation of Papers for Fault Detection is Round Trip Delay Time Measurement of Path Wireless Sensor Networks" International Journal of Advanced Research in Electronics and Communication Engineering (IJARECE) Volume 4, Issue 2, February 2015

[4]. Jin Wang, Zhongqi Zhang, Feng Xia, Weiwei Yuan and Sungyoung Lee," An Energy Efficient Stable Election-Based Routing Algorithm for Wireless Sensor Networks" sensors ISSN 1424-8220 www.mdpi.com/journal/sensors.

[5]. Hussein Mohammed Salman," Survey of routing protocols in wireless sensor networks" International Journal of Sensors and Sensor Networks, Published online January 20, 2014.

\section{BIOGRAPHY}

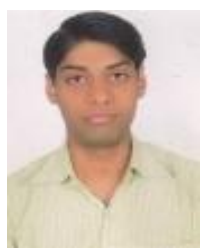

Surendra Verma is currently a research scholar of Mewar University, Chittorgarh Rajasthan (India). $\mathrm{He}$ is working with wireless sensor networking field since from 2007. He received his Master of Engineering degree in Electronics Engineering from Institute of Engineering and Technology - Devi Ahilya Vishwavidhyalay Indore (MP) India 